\title{
Crystal nucleation in sedimenting colloidal suspensions
}

Stefania Ketzetzi, John Russo, and Daniel Bonn

Citation: The Journal of Chemical Physics 148, 064901 (2018); doi: 10.1063/1.4990101

View online: https://doi.org/10.1063/1.4990101

View Table of Contents: http://aip.scitation.org/toc/jcp/148/6

Published by the American Institute of Physics

\section{Articles you may be interested in}

Structural relaxation and highly viscous flow

The Journal of Chemical Physics 148, 064502 (2018); 10.1063/1.5017218

Mobility and settling rate of agglomerates of polydisperse nanoparticles

The Journal of Chemical Physics 148, 064703 (2018); 10.1063/1.5012037

Vitrification and gelation in sticky spheres

The Journal of Chemical Physics 148, 044501 (2018); 10.1063/1.5000263

Pressure control in interfacial systems: Atomistic simulations of vapor nucleation

The Journal of Chemical Physics 148, 064706 (2018); 10.1063/1.5011106

Nanobubbles in confined solution: Generation, contact angle, and stability The Journal of Chemical Physics 148, 064704 (2018); 10.1063/1.5010991

Nanoscale hydrodynamics near solids

The Journal of Chemical Physics 148, 064107 (2018); 10.1063/1.5010401

\section{AIP | The Jounal of Chemical Physics}




\title{
Crystal nucleation in sedimenting colloidal suspensions
}

\author{
Stefania Ketzetzi, ${ }^{1, a)}$ John Russo, ${ }^{2}$ and Daniel Bonn ${ }^{1, b)}$ \\ ${ }^{1}$ Van der Waals-Zeeman Institute, Institute of Physics, Science Park 904, 1098 XH Amsterdam, The Netherlands \\ ${ }^{2}$ School of Mathematics, University of Bristol, Bristol BS8 1TW, United Kingdom
}

(Received 14 June 2017; accepted 29 January 2018; published online 13 February 2018)

\begin{abstract}
Homogeneous crystal nucleation from a metastable hard-sphere colloidal liquid has been extensively studied in simulations and experiments. A 12 order of magnitude difference between simulated and experimental nucleation rates is observed, the origin of which remains a puzzle. Here, we experimentally study crystal nucleation at the single particle level in suspensions of hard-sphere-like colloids under the influence of sedimentation. We find that sedimentation significantly enhances the nucleation rate, but contrary to what was previously thought, this is not due to simple density fluctuations, as the nucleation barriers become independent of the local density in a sedimentating fluid. Instead, we find an enhancement of the local dynamics, which suggests that local convective cells are responsible for shear-enhanced crystallization at low volume fractions. Published by AIP Publishing. https://doi.org/10.1063/1.4990101
\end{abstract}

\section{INTRODUCTION}

Homogeneous crystal nucleation from a colloidal hard sphere (HS) liquid is arguably the simplest crystallization process, encountered when small crystal nuclei form spontaneously in an existing metastable liquid phase. ${ }^{1}$ To understand crystallization kinetics, this simplest case of nucleation has received enormous attention in both simulations and experiments. For the HS fluid, the only control parameter is the volume fraction $\varphi$ occupied by the spheres. Experimentally, the HS system is reasonably well realized with suspensions of monodisperse colloidal particles that interact through a steep repulsive potential. ${ }^{2}$ In recent years, advances in imaging and particle tracking, which enabled the direct observation of crystal nucleation and growth in dense colloidal systems, ${ }^{3}$ have deepened our understanding of crystal nucleation in model HS colloids. However, an important aspect, the absolute crystal nucleation rate, remains



According to simulations, the absolute crystal nucleation rate of this system depends dramatically on $\phi$ (the effect of polydispersity and the slight softness in the interparticle potential is taken into account); a 15 order of magnitude increase of the rate is found simply going from $\phi=0.52$ to $\phi=0.54 .{ }^{5}$ This behavior is corroborated by different computational techniques. ${ }^{6,7}$ However, this behavior is not observed in experiments, where a rather weak $\varphi$ dependence is found..$^{8,9}$ The comparison between simulated and experimental rates as functions of $\varphi$ reveals a 12 order of magnitude difference for $\varphi=0.52$ that remains to be understood. Notable speculated causes for the discrepancy were a two-step crystallization

\footnotetext{
a)Present address: Huygens-Kamerlingh Onnes Laboratory, Leiden University, 2300 RA Leiden, The Netherlands.

b)Author to whom correspondence should be addressed: D.Bonn@uva.nl
}

process, ${ }^{10}$ difficulties in interpreting the experiments, ${ }^{5}$ and hydrodynamic effects, neglected in the simulations. ${ }^{6}$

One important difference between experiments and simulations is the presence of gravity, which can induce sedimentation in real life experiments, but is typically not taken into account in simulations. Despite observations that colloidal crystallization differs between micro-gravity and normal gravity experiments, how sedimentation affects the kinetics of the crystals remained unclear. ${ }^{11}$ Recently, the interplay between sedimentation and crystallization was studied via Brownian dynamics simulations. ${ }^{12}$ The findings showed that in systems influenced by sedimentation, gradients in volume fraction make that locally in the sample a high $\phi(\phi \approx 0.56)$ is reached, for which nucleation is rapid: from these regions, crystallization starts. What has to be explained is the effect of hydrodynamic interactions, not just on the crystallization process itself, but also how they couple to the gravitational field to alter the fluctuations in a suspended fluid. The role of long-range hydrodynamic interactions on the bare crystallization process remains controversial, with studies suggesting either an enhancement ${ }^{13}$ or a suppression of the crystallization rate. ${ }^{14}$

In the limit of high Peclet numbers (where gravitational effects are much stronger than thermal effects), experiments on monodisperse polystyrene particles ${ }^{15}$ have shown that longrange correlations are formed, where the amplitude of the velocity fluctuations (relative to the bare sedimentation velocity) grows with increasing packing fraction. Fluctuations are non-universal, depend strongly on the shape of the cells, ${ }^{16}$ and point to the importance of both density fluctuations and recirculations that originate locally in the sedimentating fluid. The effect of shear has also attracted a lot of interest, ${ }^{17}$ showing that the crystallization rate is a non-linear function of shear rate. ${ }^{18-20}$ Thus, within our current understanding, the nonequilibrium fluctuations that arise from the coupling between hydrodynamic interactions and the gravitational field have 
the potential to alter the crystallization process, either by an enhancement of density fluctuations or by shear-induced crystallization. In the following, we will show how to disentangle these two effects.

In this paper, we investigate experimentally what the influence of sedimentation is on crystal nucleation in HS colloids. To that end, we study both the crystallinity and the density at the single particle level by means of confocal microscopy. So far, few quantitative studies of the effect of gravity on crystal nucleation that stem from real time and space experiments have been reported. ${ }^{21}$ We find that crystal nucleation is significantly enhanced during sedimentation, irrespective of the initial volume fraction at which the system is prepared. Furthermore, we report that contrary to what was suggested before, ${ }^{12}$ the nucleation barrier does not depend significantly on the local density, showing that density fluctuations are not at the origin of the enhancement of crystal nucleation. During this regime, we observe an enhancement of the mean-square displacement of the particles, which originates from the interplay between thermal diffusion and inhomogeneous convective cells.

\section{METHODS}

We use sterically stabilized, fluorescent polymethylmethacrylate particles dispersed in a mixture of cisdecalin and cycloheptyl bromide (CHB), with $260 \mu \mathrm{M}$ tetrabutylammonium bromide salt to screen the weak electrostatic interactions between the particles. ${ }^{22}$ The hydrodynamic radius of the particles is $\mathrm{r}=0.97 \mu \mathrm{m}$, and the polydispersity in size is $2.1 \%$ (see the supplementary material). Details on the particle synthesis and the phase behavior of the particles can be found in Ref. 23. We mix cis-decalin and CHB to adjust the density mismatch $\Delta \rho$ between the solvent mixture and the particles, thereby changing the buoyant mass and the Peclet number Pe that describes the relative importance of diffusion compared to sedimentation and is defined as $P e=\frac{4 \pi g \Delta \rho r^{4}}{3 k_{B} T}$, where $g$ is the gravitational acceleration, $k_{B}$ is the Boltzmann constant, and $\mathrm{T}$ is the absolute temperature. The Peclet number also gives the ratio of the particle size to the gravitational length $l_{g}$. We prepare samples with solvent densities of $1.1 \mathrm{~g} / \mathrm{ml}$ (mismatched, $\mathrm{Pe}=0.9$, viscosity $2.3 \mathrm{mPa}$ s estimated from values reported in Ref. 22). Samples with volume fractions in the range $0.52-0.56$ are prepared by diluting sediments centrifuged to random close packing; ${ }^{24}$ in this reference also, the systematic errors in volume fraction are discussed, which can be several percent. For the density matched sample (viscosity $2.217 \mathrm{mPa} \mathrm{s}$ ), the solvent mixture was carefully adjusted until there was no visual sign of sedimentation or creaming after centrifuging for $6 \mathrm{~h}$ (at $21^{\circ} \mathrm{C}$, at $3000 \mathrm{~g}$ ). The cells for microscopy are completely filled with the suspension to avoid any suspension-air interface that we found promotes heterogeneous nucleation.

We shear melt the systems by stirring them with embedded stirrer bars, which provides a reproducible initial disordered state $^{25}$ We typically monitor $\sim 55000$ particles in a $70 \times 70$ $\times 80 \mu \mathrm{m}^{3}$ volume away from the walls of the cell and more than $30 \mu \mathrm{m}$ away from its bottom to avoid boundary effects, ${ }^{26}$ the first $23 \mathrm{~h}$ after melting by performing $1 \mathrm{scan} / \mathrm{h}$. We determine the particle positions from the confocal images using standard particle locating software. ${ }^{27}$ We perform a local bond-order analysis on the particle positions, using the spherical harmonics analysis introduced by Steinhardt et al. ${ }^{28}$ and apply the criteria established by Frenkel and co-workers ${ }^{29}$ to identify crystal particles, as well as a cluster algorithm to identify individual nuclei, ${ }^{30}$ in each configuration. To avoid boundary problems in the analysis, we only consider particles that are at least $2 \mu \mathrm{m}$ away from the boundaries of the scanned volume. It is worth noting that this choice can underestimate the size of the nuclei in the case when they are partially inside and outside the imaged volumes, inducing a systematic error that equally affects all measurements. However, we anticipate that this does not have a strong effect here, since there seem to be few nuclei at the borders, when looking at the $\mathrm{x}-\mathrm{z}$ reconstructed images in time.

\section{RESULTS AND DISCUSSION}

For all systems influenced by sedimentation, we already see the appearance of crystalline seeds during the first hours after shear melting. Figure 1 shows a typical example of the crystal nuclei found in a mismatched system at different times after the beginning of the measurement that we start 20 min after melting. On the other hand, in the density matched system of volume fraction 0.52 , we waited 6 months and we still do not observe any crystallinity. Our experiments with sedimentation then give results similar to both previous experiments and the simulations with gravity included. However, if we carefully density match our system, the nucleation rate is orders of magnitude lower, and the bound that follows from not observing any nucleation during 6 months is already much closer to the original simulation without gravity. This suggests that

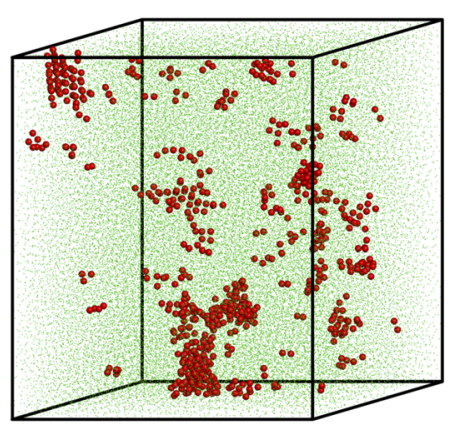

(a)



(b)
FIG. 1. Time evolution of the crystallinity in a nondensity matched system $(\mathrm{Pe}=0.9)$; the volume of the box is the same as the volume $70 \times 70 \times 80 \mu \mathrm{m}^{3}$ that we measure, far away from the walls and the bottom of the cell, which is rough to prevent heterogeneous nucleation there. Red dots correspond to the crystal particles identified (a) $1 \mathrm{~h}$ after the beginning of the measurement and (b) after $4 \mathrm{~h}$. Green dots correspond to the particles identified as liquid; their size is reduced for clarity. The arrow indicates the direction of gravity $\mathrm{g}$. 
sedimentation greatly enhances the nucleation events and may be at the origin of the earlier discrepancy between experiments and simulations.

We calculate the nucleation rate in our mismatched systems by defining the rate $\mathrm{k}$, as $k=\frac{1}{\langle t\rangle V}$, with $\langle t\rangle$ being the time we waited to find a nucleus of size 70 particles in the measured volume $\mathrm{V}$. The size is chosen as the size after which we typically observe crystalline growth in our experiments. We measured the time that passed until the appearance of a nucleus of size 70 particles in each sample. This was the biggest nucleus that we observed for the sample of volume fraction $0.52,6 \mathrm{~h}+20 \mathrm{~min}$ after melting. For the 0.54 and 0.56 samples, we observed 70 particle nucleus $1 \mathrm{~h}+20 \mathrm{~min}$ and 20 min after melting, respectively. This allows us to obtain a lower bound on the rate, which does not change significantly with the choice of this size.

In Fig. 2, we compare our nucleation rates under gravity (normalized with the short-term diffusion coefficient, $D_{0}$, and

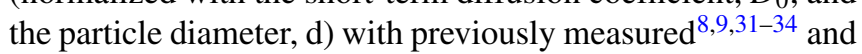
predicted $^{6,7,35}$ rates. In experiments, $D_{0}$ is typically calculated using the Stokes-Einstein formula at dilute concentrations, $D_{0}=\frac{k_{B} T}{6 \pi \eta r}$, with $\eta$ being the viscosity of the total solvent and $r$ being the hydrodynamic radius. For the density mismatched sample initially prepared at $\phi=0.52$, the rate is of the order of $10^{-8}$ in the dimensionless units of Fig. 2. For the density matched sample of $\phi=0.52$ that has not shown nucleation events so far, we calculate a bound for the rate, which corresponds to $10^{-11}$. Therefore, we find that there is at least a 3 order of magnitude difference between mismatched and matched systems in the lower liquid-crystal coexistence regime, in line with what was discussed in the Introduction. This is further supported by our experiments in a sample with a higher density mismatch (solvent density $1 \mathrm{~g} / \mathrm{ml}$ that corresponds to $\mathrm{Pe}=1.8$ ) prepared at $\phi=0.52$, where we again

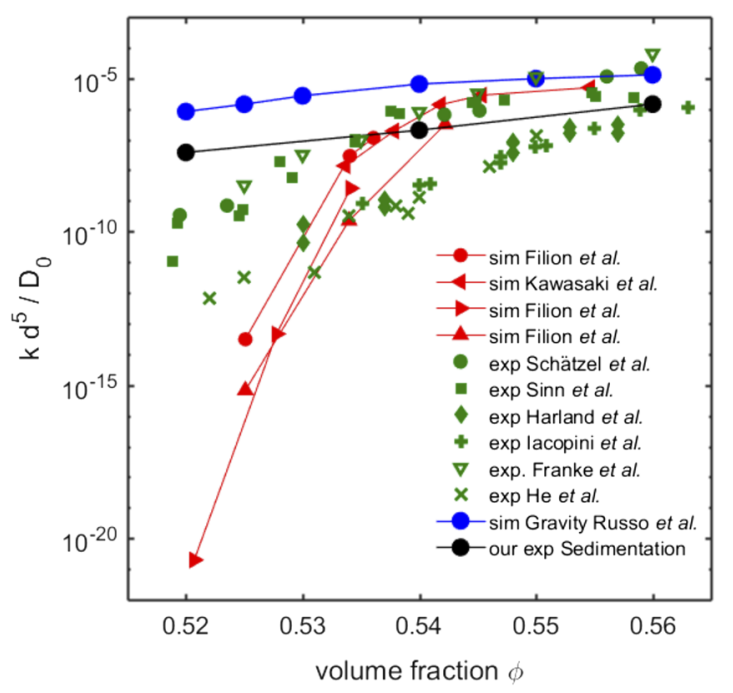

FIG. 2. Dimensionless crystal nucleation rates as a function of $\phi$ as predicted by simulations and measured in experiments. Red lines correspond to gravityfree simulations, green symbols correspond to previous experimental rates, the blue line corresponds to simulations under gravity, and the black line corresponds to systems influenced by sedimentation studied here. The Peclet number $P e=0.9$ here; it is 0.43 in Ref. 8, 0.24 in Ref. 32, and more than an order of magnitude smaller in the other experiments. observe enhanced nucleation compared to the density matched case (not shown here). Moreover, when looking at the rates from previous (light scattering) experiments, shown in green in Fig. 2, one notices roughly two trends: the upper curve where the measured rates approximate the rates predicted by the simulations with gravity in Ref. 12 and the lower curve where the measured rates are closer to the rates predicted by the gravity-free simulations in Refs. 6, 7, and 35. The data following the former stem from experiments affected by sedimentation, with Pe numbers 0.43 (Ref. 8) and 0.24 (Ref. 32), while the data following the latter stem from density matched experiments. Indeed, gravitational effects affecting previous experiments might explain the enhancement of the nucleation rate, while improved density matching already shows a decrease in the rates in the low liquid-crystal coexistence regime. To our knowledge, the only measured rates not in line with this observation are the ones stemming from the density matched experiments reported in Ref. 34, also presented in Fig. 2.

To gain insight into why nucleation is enhanced in the mismatched systems, we first look at the number of crystal nuclei, computed as the total number of nuclei detected inside the volume at each scan; we find that it increases considerably during the first hours after melting, and thereafter decreases, as shown in Fig. 3(a) (see also the supplementary material for the time evolution of both the crystalline particles and the largest nucleus size). It is worthwhile noting that these results concern all the nuclei, not just the supercritical ones. To understand how the changes in crystallinity relate to sedimentation, we first study the density order parameter $\rho_{i}$. This order parameter is calculated via Voronoi tessellation, ${ }^{30}$ which assigns each particle a local volume $v_{v o r}^{i}=\frac{1}{\rho_{i}}$ called the Voronoi volume; the Voronoi volume is then used to measure the local volume fraction, $\phi_{i}=\frac{V_{p}}{v_{v o r}^{i}}$, with $V_{p}$ being the volume of the particle. For all mismatched systems, we observe a gradual decrease of the local volume fraction with height during the first hours of the measurements and the establishment of a z-profile after $6 \mathrm{~h}$ [Fig. 3(b)], which indicates that sedimentation has stopped. Here the height is measured within the observation volume, which is kept fixed at about $30 \mu \mathrm{m}$ from the bottom of the container.

We find that crystallization is much enhanced during sedimentation and slows down when the systems have settled at longer times; after $5 \mathrm{~h}$, the density profile hardly changes anymore, and the number of nuclei starts to decrease. From the time evolution of the $2 \mathrm{D}(\mathrm{x}-\mathrm{z})$ projections of the systems, we notice that the growth of the existing nuclei is suppressed once sedimentation stops; the bigger nuclei become smaller and the small pre-critical nuclei dissolve. It should be noted that we observed qualitatively a similar behavior in samples with higher density mismatch $(\mathrm{Pe}=1.8)$. The suppression of growth once sedimentation stops has been discussed before in simulations under gravity, see Ref. 12. This already indicates that the increased nucleation probability is due to hydrodynamic effects during sedimentation, rather than changes in the local volume fraction (and consequently local changes in the supersaturation). The big and anomalous fluctuations of the z-dependent local volume fraction profiles within each time frame are also likely due to collective hydrodynamic effects; 


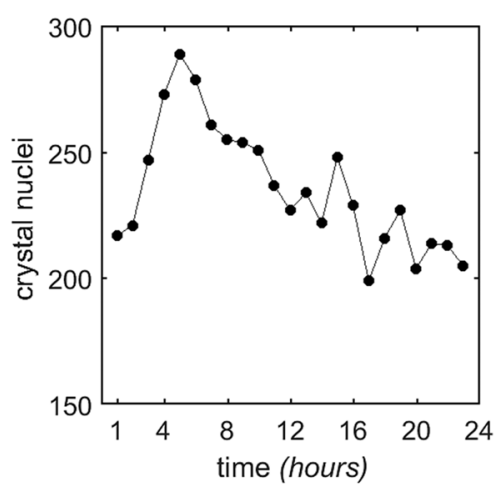

(a)

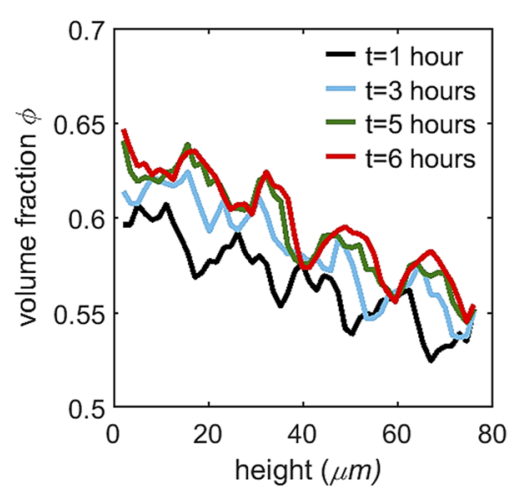

(b) it is generally invoked (in simulation studies) that at small Pe numbers $(\mathrm{Pe}<1)$ hydrodynamic interactions should be irrelevant, but there are studies that found important effects already for very low densities. ${ }^{36}$ The non-equilibrium z-dependent profiles extracted from our measurements seem to agree with the latter and probably cause convection. On the contrary, in the case of diffusive settling (sedimentation without hydrodynamics), a smooth decrease of the local volume fraction with height was observed. ${ }^{12}$ We notice that no correlation between the density inhomogeneity and the spatial distribution of crystalline nuclei is observed, as in Fig. 1.

Another, rather surprising, observation is that nucleation events happen at all heights inside the measured volumes that correspond to different volume fractions, during sedimentation. This suggests that the barrier for nucleation to occur does not significantly depend on the volume fraction, which is in sharp contrast with simulation results so far; in gravity-free simulations, the barrier was shown to drop from 40 to 20 (in $k_{B} T$ ) just going from volume fraction 0.5207 to $0.5343^{5}$ and simulations with gravity also showed that there is an optimum volume fraction $\phi \approx 0.56$ for nucleation to start. To test this hypothesis, we extract from experiments the size distribution functions of crystalline nuclei. We identify crystalline particles via bond-order parameters as described in Sec. II and compute the average volume fraction dependence of the cluster size distribution, $N_{n}$, by grouping clusters together based on their local average density, irrespective of the global volume fraction or Peclet number. Examples of $N_{n}$ are shown in the inset of Fig. 4. We average configurations at least $8 \mathrm{~h}$ after shear melting, when the density profile has settled. In the framework of Classical Nucleation Theory (CNT), the negative logarithm of the size distribution function is interpreted as an effective free energy $\left[\Delta G(n)=-k_{B} T \ln \left(N_{n}\right)\right]$. In this context, the barriers are a measure of the probability function to find a nucleus of a certain size at each local volume fraction. For a discussion on the applications of CNT to equilibrium and non-equilibrium systems, see Refs. 5 and 37, respectively. Our results for the nucleation barrier at local volume fractions in the range $0.52-0.58$ are shown as symbols in Fig. 4; to our knowledge, nucleation barriers stemming from experiments are shown here for the first time. We find that the nucleation barriers have only a weak dependence on the local volume fraction. These nucleation barriers explain the weak $\phi$ dependence and the rapid nucleation seen in previous experiments in Fig. 2.
To contrast this result with the expectations in the absence of gravity, we run Monte Carlo simulations of hard spheres at the same volume fractions considered above. To extract free-energy barriers, we use a variant of umbrella sampling, called CNT-US, which is described in detail in Ref. 38. The results are shown in Fig. 4 with dashed lines. As expected, the volume fraction dependence of the barriers is much stronger than what we find in experiments. Interestingly the experimental barriers are close to the numerical barrier computed at $\varphi=0.54$, which is the same volume fraction below which the discrepancy between simulations and experiments appears in Fig. 2.

Since the density fluctuations are not responsible for the enhanced nucleation during sedimentation, we would like to gather insight into the nature of the hydrodynamic effects; we acquire two dimensional images of our systems over a single plane. This allows us to track the particle trajectories during sedimentation in the mismatched system and compare to the trajectories in the matched system. From the trajectories, we calculate the mean squared displacement $\left\langle\delta r^{2}\right\rangle$ to quantify the particle mobility. On a log-log scale, we find a slope of 1 for the density matched system and a slope of $\frac{3}{2}$ for the density mismatched system as seen in Fig. 5. This type of super-diffusive behavior typically arises from the interplay between thermal

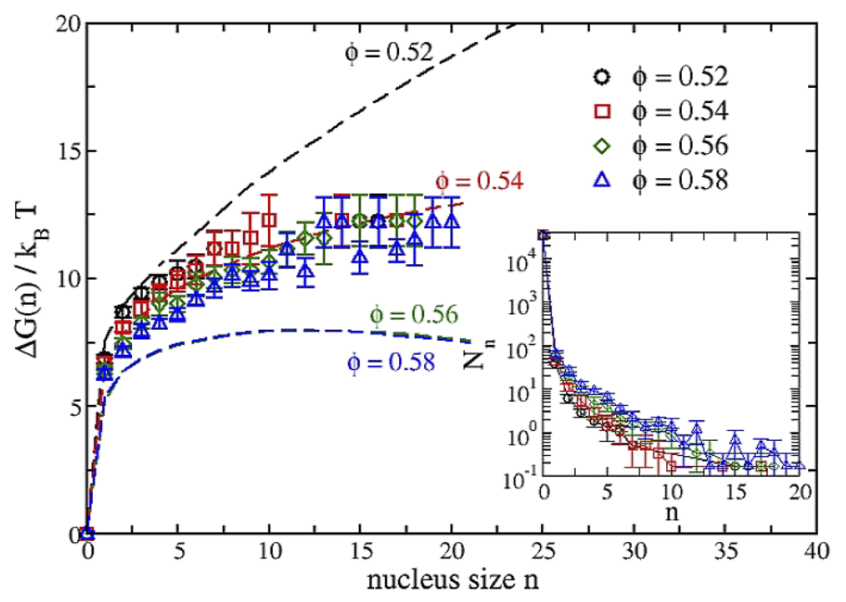

FIG. 4. Free energy in terms of thermal energy as a function of nucleus size for local volume fractions in the range 0.52-0.58 from experiments with gravity (symbols) and simulations without gravity (dashed lines). The inset shows the cluster size distribution corresponding to the free energy profiles that are shown by symbols in the main panel. 


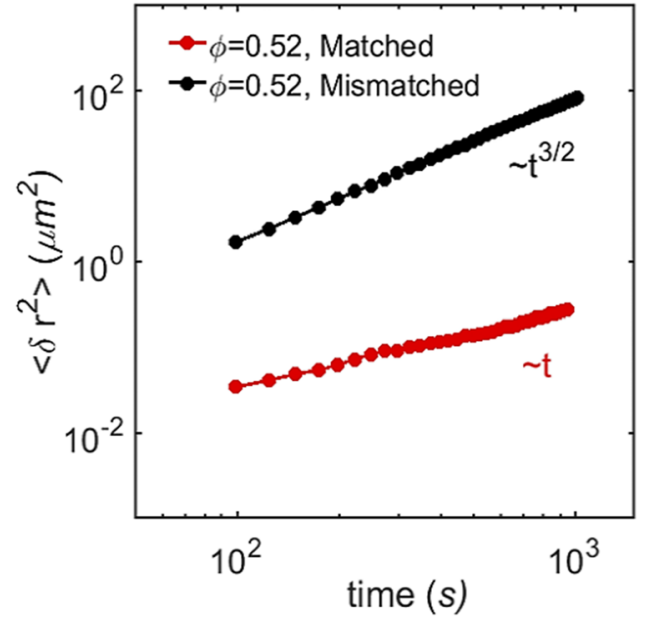

FIG. 5. Mean square displacement perpendicular to gravity as a function of time for a density mismatched system and a density matched system.

diffusion and convective cells, where spatially inhomogeneous (but correlated) velocity fields alter the power-law scaling of mass transport. ${ }^{39}$ So hydrodynamic interactions dominate the Brownian forces in our sedimenting suspensions. This in general does not come as a surprise in the case when a system is subjected to a gravitational field and was expected here already from the $\mathrm{z}$-dependent local volume fraction profiles in Fig. 3(b). It is a clear indication of flow (convection) inside the system in the direction perpendicular to gravity, which leads us to speculate that the observed enhancement of nucleation rates originates from shear-induced crystallization.

\section{CONCLUSION}

We examine crystal nucleation in HS colloids at the single particle level. We find that the sedimentation of the particles, due to the density mismatch between the particles and solvent, greatly enhances the nucleation events and the nucleation rate. In addition, we show that the nucleation barrier does not significantly depend on the local volume fraction. This greatly differs from simulation results under gravity that explain the changes in nucleation rates as being due to the changes in local volume fraction; our results rather highlight the dominant role of hydrodynamics in sedimenting suspensions.

\section{SUPPLEMENTARY MATERIAL}

See supplementary material for the determination of the size polydispersity and additional data on the crystallinity at different volume fractions as a function of time.

\section{ACKNOWLEDGMENTS}

We thank Tom Kodger for particle synthesis and useful discussions. We also thank Peter Bolhuis, Willem Kegel, and Antony Maggs for useful discussions.

${ }^{1}$ T. Palberg, "Crystallization kinetics of colloidal model suspensions: Recent achievements and new perspectives," J. Phys.: Condens. Matter 26, 333101 (2014).

${ }^{2}$ P. N. Pusey and W. van Megen, "Phase behavior of concentrated suspensions of nearly hard colloidal spheres," Nature 320, 340-342 (1986).
${ }^{3}$ U. Gasser, E. R. Weeks, A. Schofield, P. N. Pusey, and D. A. Weitz, "Realspace imaging of nucleation and growth in colloidal crystallization," Science 292, 258-261 (2001).

${ }^{4}$ G. C. Sosso, J. Chen, S. J. Cox, M. Fitzner, P. Pedevilla, A. Zen, and A. Michaelides, "Crystal nucleation in liquids: Open questions and future challenges in molecular dynamics simulations," Chem. Rev. 116, 7078-7116 (2016).

${ }^{5} \mathrm{~S}$. Auer and D. Frenkel, "Prediction of absolute crystal-nucleation rate in hard-sphere colloids," Nature 409, 1020-1023 (2001).

${ }^{6}$ L. Filion, M. Hermes, R. Ni, and M. Dijkstra, "Crystal nucleation of hard spheres using molecular dynamics, umbrella sampling and forward flux sampling: A comparison of simulation techniques," J. Chem. Phys. 133, 244115 (2010).

${ }^{7}$ L. Filion, R. Ni, D. Frenkel, and M. Dijkstra, "Simulation of nucleation in almost hard-sphere colloids: The discrepancy between experiment and simulation persists," J. Chem. Phys. 134, 134901 (2011).

${ }^{8} \mathrm{~K}$. Schätzel and B. J. Ackerson, "Density fluctuations during crystallization of colloids," Phys. Rev. E 48, 3766-3777 (1993).

${ }^{9}$ J. L. Harland and W. van Megen, "Crystallization kinetics of suspensions of hard colloidal spheres," Phys. Rev. E 55, 3054-3067 (1997).

${ }^{10}$ H. J. Schöpe, G. Bryant, and W. van Megen, "Two-step crystallization kinetics in colloidal hard-sphere systems," Phys. Rev. Lett. 96, 175701 (2006).

${ }^{11}$ J. Zhu et al., "Crystallization of hard-sphere colloids in microgravity," Nature 387, 883-885 (1997).

${ }^{12}$ J. Russo, A. C. Maggs, D. Bonn, and H. Tanaka, "The interplay of sedimentation and crystallization in hard-sphere suspensions," Soft Matter 9, 7369-7383 (2013).

${ }^{13}$ M. Radu and T. Schilling, "Solvent hydrodynamics speed up crystal nucleation in suspensions of hard spheres," Europhys. Lett. 105, 26001 (2014).

${ }^{14}$ D. Roehm, S. Kesselheim, and A. Arnold, "Hydrodynamic interactions slow down crystallization of soft colloids," Soft Matter 10, 5503-5509 (2014).

${ }^{15}$ P. N. Segre, E. Herbolzheimer, and P. M. Chaikin, "Long-range correlations in sedimentation," Phys. Rev. Lett. 79, 2574 (1997).

${ }^{16}$ S.-Y. Tee, P. J. Mucha, L. Cipelletti, S. Manley, M. P. Brenner, P. N. Segre, and D. A. Weitz, "Nonuniversal velocity fluctuations of sedimenting particles," Phys. Rev. Lett. 89, 054501 (2002).

${ }^{17}$ S. E. Paulin, B. J. Ackerson, and M. S. Wolfe, "Equilibrium and shear induced nonequilibrium phase behavior of PMMA microgel spheres," J. Colloid Interface Sci. 178, 251-262 (1996).

${ }^{18}$ R. Blaak, S. Auer, D. Frenkel, and H. Löwen, "Homogeneous nucleation of colloidal melts under the influence of shearing fields," J. Phys.: Condens. Matter 16, S3873 (2004).

${ }^{19}$ N. Koumakis, A. B. Schofield, and G. Petekidis, "Effects of shear induced crystallization on the rheology and ageing of hard sphere glasses," Soft Matter 4, 2008 (2008)

${ }^{20}$ B. Lander, U. Seifert, and T. Speck, "Crystallization in a sheared colloidal suspension," J. Chem. Phys. 138, 224907 (2013).

${ }^{21}$ F. Turci and C. P. Royall, "Crystallisation driven by sedimentation: A particle resolved study," J. Stat. Mech.: Theory Exp. 2016, 084004.

${ }^{22}$ C. P. Royall, J. Dzubiella, M. Schmidt, and A. van Blaaderen, "Nonequilibrium sedimentation of colloids on the particle scale," Phys. Rev. Lett. 98, 188304 (2007).

${ }^{23}$ T. E. Kodger, P. J. Lu, G. R. Wiseman, and D. A. Weitz, "Stable, fluorescent polymethylmethacrylate particles for the long-term observation of slow colloidal dynamics," Langmuir 33, 6382-6389 (2017).

${ }^{24}$ W. C. K. Poon et al., "On measuring colloidal volume fractions," Soft Matter 8, 21-30 (2012).

${ }^{25}$ E. R. Weeks et al., "Short- and long-range correlated motion observed in colloidal glasses and liquids," J. Phys.: Condens. Matter 19, 205131 (2007).

${ }^{26}$ R. P. A. Dullens and W. K. Kegel, "Reentrant surface melting of colloidal hard spheres," Phys. Rev. Lett. 92, 195702 (2004).

${ }^{27}$ J. C. Crocker and D. G. Grier, "Methods of digital video microscopy for colloidal studies," J. Colloid Interface Sci. 179, 298-310 (1996).

${ }^{28}$ P. J. Steinhardt et al., "Bond-orientational order in liquids and glasses," Phys. Rev. B 28, 784 (1983).

${ }^{29}$ P. R. ten Wolde et al., "Numerical calculation of the rate of crystal nucleation in a Lennard-Jones system at moderate undercooling," J. Chem. Phys. 104, 9932-9947 (1996).

${ }^{30} \mathrm{~J}$. Russo and H. Tanaka, "The microscopic pathway to crystallization in supercooled liquids,” Sci. Rep. 2, 505 (2012). 
${ }^{31}$ Y. He, B. J. Ackerson, W. van Megen, S. M. Underwood, and K. Schätzel, "Dynamics of crystallization in hard-sphere suspensions," Phys. Rev. E 54, 5286 (1996)

${ }^{32}$ C. Sinn et al., "Solidification kinetics of hard-sphere colloidal suspensions," Prog. Colloid Polym. Sci. 118, 266-275 (2001).

${ }^{33} \mathrm{~S}$. Iacopini et al., "Crystallization kinetics of polydisperse hard-spherelike microgel colloids: Ripening dominated crystal growth above melting," J. Chem. Phys. 130, 084502 (2009).

${ }^{34}$ M. Franke, S. Golde, and H. J. Schöpe, "Solidification of a colloidal hard sphere like model system approaching and crossing the glass transition," Soft Matter 10, 5380-5389 (2014).
${ }^{35}$ T. Kawasaki and H. Tanaka, "Formation of a crystal nucleus from liquid," Proc. Natl. Acad. Sci. U. S. A. 107, 14036-14041 (2010).

${ }^{36} \mathrm{~J}$. Padding and A. A. Louis, "Hydrodynamic and brownian fluctuations in sedimenting suspensions," Phys. Rev. Lett. 93, 220601 (2004).

${ }^{37}$ R. Blaak et al., "Crystal nucleation of colloidal suspensions under shear," Phys. Rev. Lett. 93, 068303 (2004).

${ }^{38}$ J. Russo, F. Romano, and H. Tanaka, "New metastable form of ice and its role in the homogeneous crystallization of water," Nat. Mater. 13, 733-739 (2014).

${ }^{39}$ J. Bouchaud et al., "Superdiffusion in random velocity fields," Phys. Rev. Lett. 64, 2503-2506 (1990). 